Enferm Bras 2020;19(1):4-10

https://doi.org/10.33233/eb.v19i1.3977

\title{
ARTIGO ORIGINAL \\ O saber acadêmico sobre as competências do enfermeiro na prevenção do câncer do colo do útero
}

\author{
Paloma Cavalcante de Assis Martins*, Ilse Sodré da Motta, D.Sc.** \\ *Enfermeira Residente do Programa de Residência Multiprofissional "Atenção ao Paciente Adulto \\ Neurocirúrgico em UTI" pelo Hospital Universitário Getúlio Vargas, Universidade Federal do \\ Amazonas, ${ }^{*} U N E S P$, Campus Botucatu, Obstetrícia e Mastologia
}

Recebido em 24 de outubro de 2018; aceito em 21 de janeiro 2020.

Correspondência: Paloma Cavalcante de Assis Martins, Avenida Apurinã, 4, Praça 14 de Janeiro, 69020-170 Manaus AM

Paloma Cavalcante de Assis Martins: palomassis@yahoo.com.br Ilse Sodré da Motta: ismotta@ufam.edu.br

\section{Resumo}

No Brasil, o câncer de colo do útero é o quarto tipo de câncer mais comum entre as mulheres, sendo estimados, para 2018, dezesseis mil novos casos. Objetivou-se identificar o saber teórico de acadêmicos concluintes do Curso de Enfermagem, quanto às competências do enfermeiro durante a consulta de enfermagem sobre o exame preventivo do câncer do colo do útero (PCCU). Estudo de abordagem quantitativa realizado com 30 acadêmicos concluintes, em outubro de 2017. Os dados foram produzidos por meio de questionário com perguntas objetivas, analisados através de procedimentos estatísticos e organizados em forma de tabelas. Verificou-se que o nível de conhecimento dos acadêmicos acerca do tema é de $64 \%$. As questões com menor quantidade de acertos dizem respeito à faixa etária de mulheres que deve ser priorizada para 0 PCCU, rotina de rastreamento, recomendações prévias para a realização do exame preventivo, atendimento precedente à coleta do exame e avaliação do resultado do mesmo. O estudo evidenciou que há fragilidades no saber teórico dos acadêmicos quanto às competências do enfermeiro durante a consulta de enfermagem no PCCU. Desse modo, espera-se que este estudo possa contribuir com a formação de futuros enfermeiros e a adequação do ensino de enfermagem na área de saúde da mulher.

Palavras-chave: enfermeiro, prevenção, câncer de colo do útero.

\begin{abstract}
The academic knowledge about nursing care in the prevention of cervical cancer

In Brazil, cervical cancer is the fourth cancer most common among women, being estimated, for 2018, sixteen thousand new cases. The objective was to identify the theoretical knowledge of nursing graduating academics about nursing care in the prevention of cervical cancer. It is a quantitative study with 30 graduating academics carried out in October 2017. Data were generated through open-ended questionnaire, analyzed through statistical procedures and organized in the form of spreadsheet. The level of knowledge of academics about the subject is $64 \%$. The questions with fewer hits are about the age group of women that must be prioritized in the prevention of cervical cancer, screening routine, previous recommendations for the examination, prior attendance to exam collection and outcome assessment. The study showed there are frailties in the theoretical knowledge of academics about nursing care in the prevention of cervical cancer. Thereby, it is expected this study contribute with the professional qualification of new nurses and the adequacy of the nursing education in the field of women's health.
\end{abstract}

Keywords: nurse, prevention, uterine cervical neoplasms. 


\section{Resumen \\ Saber teórico de académicos sobre las competencias del enfermero en la prevención de cáncer de cuello de útero}

En Brasil, el cáncer de cuello de útero es el cuarto tipo de cáncer más común entre las mujeres, siendo estimados, para 2018, dieciséis mil nuevos casos. Se objetivó identificar el saber teórico de académicos concluyentes del Curso de Enfermería, en cuanto a las competencias del enfermero en la prevención de este cáncer. Estudio de abordaje cuantitativo realizado con 30 académicos concluyentes, en octubre de 2017. Los datos fueron producidos por medio de cuestionario con preguntas objetivas, analizados a través de procedimientos estadísticos y organizados en forma de tablas. Se verificó que el nivel de conocimiento de los académicos es $64 \%$. Las cuestiones con menor cantidad de aciertos se refieren al grupo de edad de mujeres que deben ser priorizadas, rutina de rastreo, recomendaciones previas para la realización del examen, atención previa a la recolección y evaluación del resultado. El estudio evidenció que hay fragilidades en el saber teórico de los académicos en cuanto a las competencias del enfermero en la prevención del cáncer de cuello de útero. De este modo, se espera que este estudio pueda contribuir con la formación de futuros enfermeros y la adecuación de la enseñanza de enfermería en el área de salud de la mujer.

Palabras-clave: enfermero, prevención, neoplasias del cuello uterino.

Introdução

No Brasil, o Câncer de Colo do Útero (CCU), também chamado de câncer cervical, é o quarto tipo de câncer mais comum entre as mulheres. Com exceção do câncer de pele, esse tumor é o que apresenta maior potencial de prevenção e cura quando diagnosticado precocemente. Só para o ano de 2018 foram estimados cerca de dezesseis mil novos casos [1].

$\mathrm{Na}$ análise regional do Brasil, o CCU destaca-se como o primeiro mais incidente na região norte, com 23,97 casos por 100 mil mulheres. A região centro-oeste ocupa a segunda posição $(20,72 / 100 \mathrm{mil})$, a nordeste a terceira (19,49/100 mil), a quarta mais incidente é a região sul (15,17/100 mil) e a quinta a região sudeste (11,3/100 mil) [2].

Esse tema se insere no âmbito da saúde da mulher, área considerada estratégica para ações prioritárias no Sistema Único de Saúde (SUS) no nível da Atenção Básica (AB). A concentração de esforços governamentais aliada à produção acadêmica e a atuação dos profissionais trouxe melhorias no acesso à prevenção do CCU em todo o país. Entretanto, ainda se mostra insuficiente como sinalizado nas estimativas de incidência e, em muitas regiões e situações, o diagnóstico ainda é feito em estágios avançados da doença [2,3].

É atribuição da $A B$, através da Estratégia de Saúde da Família (ESF), prestar cuidado integral e conduzir ações de promoção à saúde, rastreamento e detecção precoce, bem como acompanhar o seguimento terapêutico das mulheres nos demais níveis de atenção, quando diante de resultado de exame Preventivo do Câncer de Colo do Útero (PCCU) alterado [4].

Nesse contexto, o enfermeiro é o profissional imprescindível na ESF, capacitado a exercer atividades técnicas específicas de sua competência, administrativas e educativas e através do vínculo com as usuárias, concentrar esforços para reduzir os tabus, mitos e preconceitos, buscando o convencimento da clientela feminina sobre os benefícios da prevenção $[3,4]$.

A capacidade do enfermeiro em exercer o trabalho de forma humanizada e integral, além de identificar as necessidades de saúde da população, deve ser adquirida ao longo da graduação e oferecida pela instituição em que foi formado. Normalmente, a formação de um profissional para atender ao perfil que o mercado de trabalho exige pode estar relacionada ao método adotado no projeto pedagógico do curso [5]. No Brasil, o processo de formação do enfermeiro foi regulamentado pela Resolução n.03, de 07/11/2001, e aponta que este profissional deve receber uma formação crítico-reflexiva para intervir nos problemas de atenção à saúde da população [6].

Paulo Freire, um dos precursores da educação problematizadora, defende a prática educativo-progressiva em favor da autonomia dos discentes. Nessa perspectiva, esses atores sociais têm participação ativa no processo de ensino-aprendizagem, sendo um agente crítico, participante e construtor do conhecimento, em que o docente é apenas o facilitador do aprendizado [7].

Assim, o enfermeiro que possui essa capacidade de reflexão torna-se capaz de desenvolver uma assistência de qualidade, holística, conforme a necessidade de cada mulher [5]. É fundamental que esse profissional durante as consultas de enfermagem do PCCU, ofereça 
uma assistência com olhar integral, sempre pensando que a mulher que está diante de si, pode estar exposta e vulnerável a desenvolver a doença [8].

Diante dessa problemática, objetivou-se identificar o saber teórico de acadêmicos concluintes do Curso de Enfermagem de uma universidade localizada na cidade de Manaus, quanto às competências do enfermeiro da $A B$ durante a consulta de enfermagem no PCCU.

\section{Material e métodos}

Trata-se de um estudo quantitativo, com caráter descritivo-exploratório, desenvolvido em uma universidade localizada na cidade de Manaus, com acadêmicos concluintes do Curso de Enfermagem, no período do mês de outubro de 2017.

Teve como sujeitos 30 acadêmicos, identificados no estudo com nomes fictícios, resguardando o anonimato, que estavam regularmente matriculados e que concordaram em participar do estudo, sendo excluídos aqueles que estavam de licença médica, licença maternidade ou em atividade militar.

A coleta de dados foi realizada após assinatura do Termo de Consentimento Livre e Esclarecido pelos sujeitos e aprovação do Comitê de Ética em Pesquisa da Universidade Federal do Amazonas.

Para a produção dos dados foi utilizada a técnica de questionário com 10 perguntas objetivas, tendo a finalidade de atender especificamente ao objetivo desta pesquisa e abordando aspectos como: fatores de risco do câncer de colo do útero, manifestações clínicas da doença, faixa etária de abrangência do exame preventivo, rotina de rastreamento, situações indicadas para a coleta do exame, recomendações prévias para a realização do mesmo, abordagem à paciente, etapas e resultados do exame, além da importância do profissional enfermeiro como agente atuante na prevenção desse câncer (Apêndice A).

Pesquisas de natureza quantitativa consideram que a realidade só pode ser compreendida com base na análise de dados brutos, recorrendo à linguagem matemática para descrever as causas de um fenômeno, as relações entre variáveis, etc. [9]. Através da enfatização da objetividade, analisa os dados numéricos através de procedimentos estatísticos [10].

Com base nisso, as respostas dos questionários foram tabeladas de forma a serem analisadas e contabilizadas estatisticamente. A partir daí, a porcentagem dos acertos e erros dos participantes a cada questão do instrumento foi disposta em tabelas com o intuito de quantificar os dados. Baseando-se na análise desses dados brutos, pôde-se utilizar a linguagem matemática para buscar conclusões a respeito do saber teórico dos acadêmicos concluintes sobre as habilidades do enfermeiro na assistência preventiva à saúde da mulher durante a consulta de PCCU.

\section{Resultados e discussão}

O nível de conhecimento dos 30 acadêmicos concluintes, participantes do estudo, acerca do tema é de $64 \%$ (Tabela I). Em relação à quantidade de acertos por acadêmico, $50 \%$ deles acertaram entre 6 e 8 questões, enquanto $6 \%$ acertaram entre 2 e 4 questões. Nenhum dos 30 acadêmicos concluintes acertou todas as questões do instrumento, assim como nenhum deles obteve nota zero (Tabela II e Figura 1).

Tabela I - Total de acertos e nível de conhecimento acerca do tema dos 30 acadêmicos concluintes que participaram do estudo.

\begin{tabular}{ll}
\hline Total de acertos & Nivel de conhecimento \\
\hline 193 & $64 \%$ \\
\hline
\end{tabular}

Dividiu-se o número total de acertos (193) pelo número total de questões (30 questionários x 10 questões: 300 questões), obtendo valor igual a $0,64-64 \%$ de nível de conhecimento.

Tabela II - Índice geral de desempenho dos 30 acadêmicos concluintes, participantes do estudo. 


\begin{tabular}{lllll}
\hline Acertos & FA $^{1}$ & FR $^{2}$ & FAA $^{3}$ & FRA $^{4}$ \\
\hline $0-2$ & 0 & $0 \%$ & 0 & $0 \%$ \\
$2-4$ & 2 & $6 \%$ & 2 & $6 \%$ \\
$4-6$ & 5 & $17 \%$ & 7 & $23 \%$ \\
$6-8$ & 15 & $50 \%$ & 22 & $73 \%$ \\
$8-10$ & 8 & $27 \%$ & 30 & $100 \%$ \\
\hline Total & 30 & $100 \%$ & & \\
\hline
\end{tabular}

${ }^{1}$ Frequência absoluta; ${ }^{2}$ Frequência relativa; ${ }^{3}$ Frequência absoluta acumulada; ${ }^{4}$ Frequência relativa acumulada. Dividiu-se o número de acadêmicos que tiveram certa quantidade de acertos pelo número total de participantes (30).

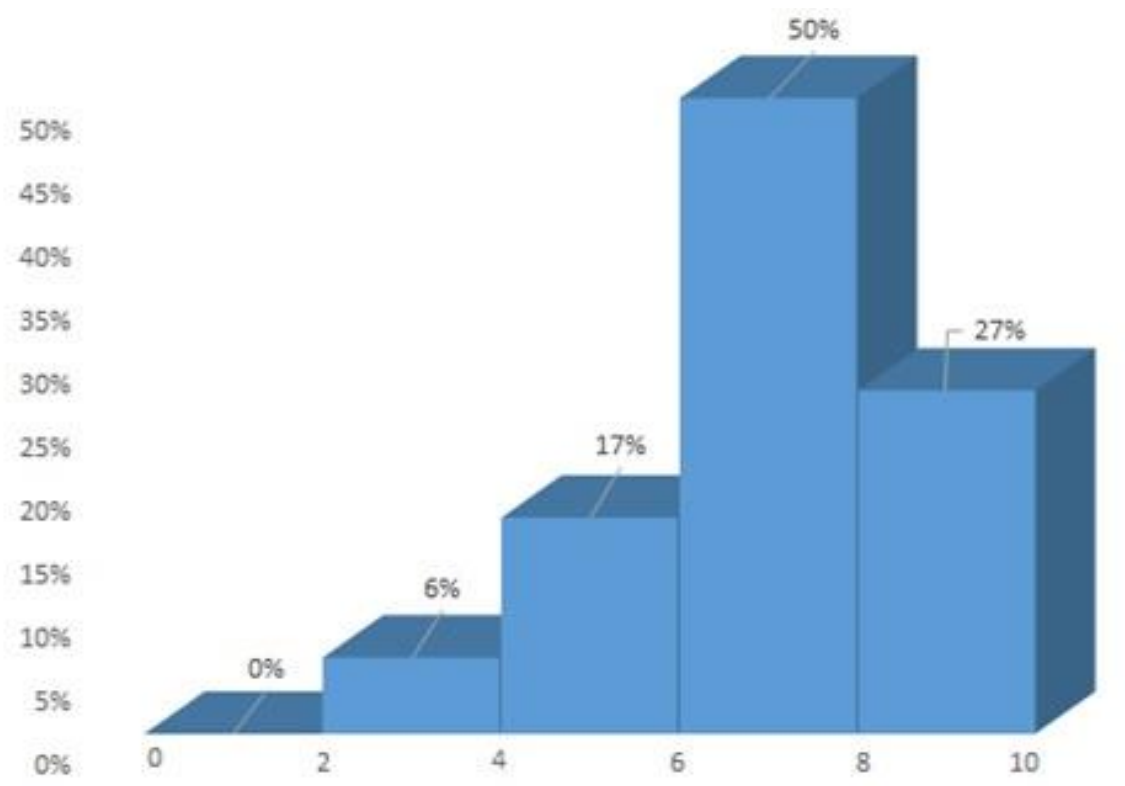

Figura 1 - Frequência relativa (porcentagem) de acertos por classes.

Observa-se que $50 \%$ dos acadêmicos acertaram entre 6 e 8 questões, enquanto $6 \%$ acertaram entre 2 e 4 questões.

Tabela III - Quantidade e porcentagem de acertos por questão.

\begin{tabular}{ccc}
\hline Questão & Acertos & Porcentagem \\
\hline 1 & 23 & $77 \%$ \\
\hline 2 & 25 & $83 \%$ \\
\hline 3 & 17 & $56 \%$ \\
\hline 4 & 13 & $43 \%$ \\
\hline 5 & 25 & $83 \%$ \\
\hline 6 & 5 & $17 \%$ \\
\hline 7 & 19 & $63 \%$ \\
\hline 8 & 21 & $70 \%$ \\
\hline 9 & 16 & $53 \%$ \\
\hline 10 & 29 & $97 \%$
\end{tabular}

Dividiu-se o número total de acertos de cada questão pelo número total de acadêmicos concluintes (30).

Analisando a quantidade de acertos por questão, o desempenho dos acadêmicos variou bastante. É possível observar que determinadas questões obtiveram índice de acertos acima de $70 \%$, enquanto outras este índice ficou abaixo de 50\% (Tabela III).

A primeira questão, relacionada aos fatores de risco associados ao câncer de colo do útero, como uso prolongado de contraceptivos orais e tabagismo, obteve 23 acertos $(77 \%)$. Notou-se que a maioria dos acadêmicos conseguiu identificar os fatores de risco da doença corretamente.

A segunda questão discorre sobre as manifestações clínicas da doença, como sangramento vaginal, leucorreia e dor pélvica, além de tumoração e necrose do colo do útero durante o exame especular. Com 25 acertos (83\%), observou-se que grande parte dos acadêmicos considerou corretamente as opções citadas [1,4]. 
A terceira questão diz respeito à faixa etária de mulheres que deve ser priorizada durante a realização do exame preventivo. Com 17 acertos (56\%), percebeu-se dificuldade dos acadêmicos em responder a alternativa correta (25 a 64 anos). Esse equívoco é compreensível, pois na realidade as meninas estão entrando na vida sexualmente ativa cada vez mais cedo. Essa sexualidade precoce torna necessário que o exame preventivo seja realizado antes da faixa etária recomendada pelo Ministério da Saúde.

A quarta questão, relacionada à rotina de rastreamento do exame preventivo - após dois exames normais consecutivos com intervalo de um ano, deve-se repetir o exame somente três anos depois, obteve 13 acertos (43\%). Notou-se que mais da metade dos acadêmicos analisados desconhece a rotina do exame $[1,4]$.

A quinta questão discorre sobre as situações em que o exame preventivo é indicado, trazendo as seguintes opções corretas: gestantes e mulheres na pós-menopausa devem continuar a rotina de rastreamento, mesmo estando em situações diferenciadas. Com 25 acertos (83\%), observou-se que grande parte dos acadêmicos considerou corretamente as opções citadas.

A sexta questão diz respeito às recomendações prévias para a realização do exame preventivo. Com apenas 5 acertos (17\%), essa questão foi a que obteve o menor índice de respostas corretas. Percebeu-se que os acadêmicos desconhecem que a abstinência sexual prévia ao exame só é justificada quando são utilizados preservativos com lubrificantes ou espermicidas. Na prática a presença de espermatozoides não compromete a avaliação microscópica $[1,4]$.

A sétima questão, relacionada às etapas do atendimento prévio à coleta do exame: identificação da paciente, explicação de como é realizado o preventivo, investigação da história clínica da cliente, preenchimento dos dados no formulário para requisição do exame, preparo da lâmina e, por último, solicitar à mulher para esvaziar a bexiga e trocar a roupa por um avental, obteve 19 acertos (63\%). Notou-se que a maioria dos acadêmicos conhece o passo a passo do atendimento prévio à realização do exame.

A oitava questão discorre sobre o procedimento de coleta do exame preventivo. Com 21 acertos (70\%), observou-se que grande parte dos acadêmicos conhece como proceder durante a coleta em caso de mulheres idosas com vagina atrófica. Também demonstraram conhecimento em como coletar material citológico da ectocérvice e da endocérvice e qual o melhor material utilizado para fixar o esfregaço $[1,4]$.

A nona questão diz respeito ao resultado do exame preventivo. Com 16 acertos (53\%), percebeu-se certa dificuldade por parte dos acadêmicos em responder a questão corretamente, praticamente a metade desconhece os achados microbiológicos considerados normais e os níveis de Neoplasia Intra-Epitelial Cervical. A décima questão, relacionada às atribuições do enfermeiro no que tange a prevenção do câncer de colo do útero, obteve 29 acertos (97\%). Notou-se que quase todos os acadêmicos participantes do estudo reconhecem essas atribuições, que vão desde a busca ativa às mulheres para a coleta do exame e a entrega do resultado, até a realização da consulta de enfermagem de maneira integral, buscando sinais e sintomas de outras Infecções Sexualmente Transmissíveis e tratando-as adequadamente, não focando apenas no câncer de colo do útero [1,4].

O estudo evidenciou que há fragilidades no saber teórico dos acadêmicos concluintes do Curso de Enfermagem sobre as competências do enfermeiro na consulta durante o Preventivo do Câncer de Colo do Útero. Os tópicos nos quais os acadêmicos demonstraram maior dificuldade em responder as questões corretamente - índice abaixo de $70 \%$ de aproveitamento - foram a respeito da faixa etária de mulheres que deve ser priorizada para o PCCU, rotina de rastreamento, recomendações prévias para a realização do exame preventivo, atendimento precedente à coleta do exame e avaliação do resultado do mesmo.

Porém, nota-se também que os acadêmicos obtiveram resultados satisfatórios em outros pontos abordados no questionário. Podem-se citar a avaliação de fatores de risco para o câncer de colo do útero, manifestações clínicas da doença, situações em que o exame preventivo é ou não indicado, como coletar o exame e, principalmente, as atribuições e a importância do profissional enfermeiro na deteç̧ão precoce do câncer de colo do útero.

Desse modo, espera-se que este estudo possa contribuir para a reflexão sobre a formação do enfermeiro e colabore para a adequação do ensino de enfermagem na área de saúde da mulher. Uma possível adequação do ensino possibilitará em uma melhor oferta aos 
serviços de saúde de profissionais capacitados em detectar precocemente o câncer de colo do útero. Além de aumentar a quantidade de pesquisas científicas brasileiras no campo de oncologia, especificamente nesse tipo de câncer, contribuindo, assim, para alcançar uma assistência de melhor qualidade à população.

\section{Referências}

1. Brasil. Ministério da Saúde. Instituto Sírio-Libanês de Ensino e Pesquisa. Protocolos da atenção básica: saúde das mulheres. Brasília (DF): Ministério da Saúde; 2016. 230 p.

2. Instituto Nacional do Câncer (Brasil). Estimativa 2016. Incidência do câncer no Brasil. Rio de Janeiro: INCA; 2015.

3. Melo MCSC, Vilela F, Salimena AMO, Souza IEO. O enfermeiro na prevenção do câncer do colo do útero: o cotidiano da atenção primária. Rev Bras Cancerol 2012;58(3):389-98.

4. Brasil. Ministério da Saúde. Secretaria de Atenção à Saúde. Departamento de Atenção Básica. Controle dos cânceres do colo do útero e da mama. Brasília (DF): Ministério da Saúde; 2013. 124 p. (Cadernos de Atenção Básica, oㅡ 13); (Série A. Normas e manuais técnicos).

5. Viana MRP, Moura MEB, Nunes BMVT, Monteiro CFS, Lago EC. Formação do enfermeiro para a prevenção do câncer de colo uterino. Rev Enferm UERJ 2013;21 (1):624-30.

6. Brasil. Ministério da Educação. Conselho Nacional de Educação. Câmara da Educação Superior. Resolução no 3, de 07 de novembro de 2001. Institui Diretrizes Curriculares Nacionais do Curso de Graduação em Enfermagem. Brasília (DF): Conselho Nacional de Educação; 2001.

7. Freire P. Pedagogia da autonomia: saberes necessários à prática educativa. $37^{a} \mathrm{ed}$. São Paulo (SP): Paz e Terra; 2008.

8. Brasil. Ministério da Saúde. Política Nacional de Atenção Básica. Brasília: MS; 2016.

9. Fonseca JJS. Metodologia da pesquisa científica. Apostila. Fortaleza (CE): UEC; 2002.

10. Polit DF, Beck CT, Hungler BP. Fundamentos de pesquisa em enfermagem: métodos, avaliação e utilização. Trad. de Ana Thorell. 5ª ed. Porto Alegre (RS): Artmed; 2004.

\section{Apêndice A - Instrumento de coleta de dados - Questionário*}

Seguem abaixo questões sobre os saberes teóricos do enfermeiro na consulta de enfermagem durante o exame Preventivo do Câncer de Colo do Útero (PCCU). Marcar um "X" na resposta de sua preferência:

1) Qual das seguintes alternativas abaixo NÃO caracteriza um fator de risco associado ao câncer de colo do útero?
a. ( ) Uso prolongado de contraceptivos orais.
b. ( ) Tabagismo.
c. ( ) Idade tardia da atividade sexual.

\section{2) Em relação às manifestações clínicas do câncer de colo do útero, marque a opção} FALSA:

a. ( ) No estágio invasor da doença os principais sintomas são sangramento vaginal, leucorreia e dor pélvica.

b. ( ) As lesões precursoras do câncer de colo do útero são sintomáticas.

c. ( ) Ao exame especular, podem ser evidenciados sangramento, tumoração, ulceração e necrose no colo do útero.

3) A realização do PCCU deve priorizar as mulheres de qual faixa etária?

a. ( ) 25 a 64 anos.

b. ( ) 25 a 60 anos.

c. ( ) 20 a 64 anos.

4) A respeito da rotina de rastreamento do PCCU, complete a frase: 
"A rotina recomendada para o rastreamento no Brasil é a repetição do PCCU a cada anos, após exam.
a. ( ) 2,2 e 1.
b. ( ) 3,2 e 1 .
c. ( ) 3,3 e 1 .

5) Em qual das seguintes situações abaixo a coleta do PCCU NÃO é indicada?

a. ( ) Mulheres sem história de atividade sexual.

b. ( ) Gestantes.

c. ( ) Mulheres na pós-menopausa.

6) Em relação às recomendações prévias para a realização do exame, marque a opção FALSA:

a. ( ) Embora usual, a recomendação de abstinência sexual prévia ao exame só é justificada quando são utilizados preservativos com lubrificantes ou espermicidas.

b. ( ) No caso de sangramento vaginal anormal, o exame ginecológico não é mandatório.

c. ( ) O exame não deve ser feito no período menstrual, pois a presença de sangue pode prejudicar o diagnóstico citopatológico.

7) Qual a sequência correta das etapas do atendimento prévias à coleta?

1. Informar a paciente sobre o propósito do exame e as etapas do procedimento.

2. Realizar a identificação: checar nome, data de nascimento, endereço.

3. Preencher os dados nos formulários para requisição do PCCU.

4. Solicitar que a mulher esvazie a bexiga e troque a roupa, em local reservado, por um avental ou camisola.

5. Preparar a lâmina.

6. Buscar a história clínica da paciente.

a. ( ) $1,2,3,4,5$ e 6 .

b. ( ) $3,6,2,1,4,5$.

c. ( ) $2,1,6,3,5,4$.

8) Em relação ao procedimento da coleta do PCCU, marque a opção VERDADEIRA:

a. ( ) Não utilizar o espéculo com lubrificante, mas em casos de mulheres idosas com vagina atróficas, recomenda-se molhar o espéculo com soro fisiológico.

b. ( ) Para coleta da endocérvice utiliza-se a espátula de Ayre, do lado que apresenta reentrância.

c. ( ) A melhor maneira para fixar o esfregaço citológico é utilizando álcool a 100\%.

9) Em relação ao resultado do PCCU, marque a opção FALSA:

a. ( ) Resultados indicando achados microbiológicos (lactobacillussp, cocos e outros bacilos) são considerados normais.

b. ( ) NIC (Neoplasia Intra-Epitelial Cervical) 1 indica displasia leve.

c. ( ) O acrônimo ASCUS (Células Escamosas Atípicas de Significado Indeterminado) indica sinal claro de que há uma alteração pré-maligna.

10) Considerando as competências do enfermeiro como agente atuante na detecção precoce do câncer de colo do útero, marque a opção FALSA:

a. ( ) É atribuição do enfermeiro aguardar a busca passiva das mulheres pelo resultado do PCCU, descartando aqueles resultados que não obtiveram procura.

b. ( ) É atribuição do enfermeiro orientar as mulheres com resultado de exame normal a seguir a rotina de rastreamento.

c. ( ) É atribuição do enfermeiro realizar consulta de maneira integral, observando sinais e sintomas de outras Infecções Sexualmente Transmissíveis e tratando-as adequadamente, não focando apenas no câncer de colo do útero.

${ }^{*}$ Questões elaboradas a partir das seguintes referências:

1. Brasil. Ministério da Saúde. Instituto Sírio-Libanês de Ensino e Pesquisa. Protocolos da atenção básica: saúde das mulheres. Brasília (DF): Ministério da Saúde; 2016. 230 p.

2. Brasil. Ministério da Saúde. Secretaria de Atenção à Saúde. Departamento de Atenção Básica. Controle dos cânceres do colo do útero e da mama. Brasília (DF): Ministério da Saúde; 2013. 124p. (Cadernos de Atenção Básica, ํํ13); (Série A. Normas e manuais técnicos). 